\title{
A transpedicular approach to complex ventrally situated thoracic intradural extramedullary tumors: technique, indications, and multiinstitutional case series
}

\author{
Zachary R. Visco, BSE, ${ }^{1}$ David D. Liu, BS, ${ }^{2}$ Owen P. Leary, BS, ${ }^{2}$ Adetokunbo A. Oyelese, MD, PhD, ${ }^{2}$ \\ Ziya L. Gokaslan, MD, ${ }^{2}$ Joaquin Q. Camara-Quintana, MD, ${ }^{2}$ and Michael A. Galgano, MD' \\ 'Department of Neurosurgery, State University of New York, Upstate Medical University, Syracuse, New York; and 2Department \\ of Neurosurgery, Rhode Island Hospital, The Warren Alpert Medical School of Brown University, Providence, Rhode Island
}

\begin{abstract}
OBJECTIVE Ventrally situated thoracic intradural extramedullary tumors are surgically challenging and difficult to access, and they may be complicated by extensive adhesions and calcifications. Selecting an approach for adequate ventral access is key to complete resection and optimization of outcomes. The authors present a case series of patients who underwent resection of ventral thoracic intradural extramedullary tumors and discuss indications and considerations for this technique. Additionally, they describe the use of a posterolateral transpedicular approach for resection of ventral thoracic intradural extramedullary tumors compared with other techniques, and they summarize the literature supporting its application.
\end{abstract}

METHODS From May 2017 to August 2020, 5 patients with ventral thoracic intradural extramedullary tumors underwent resection at one of the two academic institutions.

RESULTS Patient ages ranged from 47 to 75 (mean 63.4) years. All tumors were diagnosed as meningiomas or schwannomas by histological examination. Three of the 5 patients had evidence of partial or extensive tumor calcification. Four of the 5 patients underwent an initial posterolateral transpedicular approach for resection, with positive radiographic and clinical outcomes from surgery. One patient initially underwent an unsuccessful traditional direct posterior approach and required additional resection 2 years later after interval disease progression. There were no postoperative wound infections, CSF leaks, or other complications related to the transpedicular approach.

CONCLUSIONS Posterolateral transpedicular tumor resection is a safe technique for the treatment of complex ventrally situated thoracic intradural extramedullary tumors compared with the direct posterior approach. Anecdotally, this approach appears to be particularly beneficial in patients with calcified tumors.

https://thejns.org/doi/abs/10.3171/2021.2.FOCUS20968

KEYWORDS posterolateral; transpedicular; ventral intradural extramedullary tumors

$\mathrm{S}$ PINAL meningiomas and schwannomas account for roughly two-thirds of all spinal tumors, and their resection presents a surgical challenge. ${ }^{1}$ They are predominantly found in the thoracic spine, where the spinal canal is particularly narrow. Accordingly, attempts at thoracic intradural tumor debulking risk excessive spinal cord traction and the need for spinal cord manipulation. ${ }^{2,3} \mathrm{~A}$ subset of thoracic intradural tumors are ventrally situated, which makes primary access even more difficult because the surgical corridor is obstructed by the cord posteriorly, the mediastinum anteriorly, and the paravertebral musculature laterally. ${ }^{2}$ Given the difficulty of surgical access, ventral location is a negative prognostic indicator. ${ }^{3}$ Alternative surgical approaches for thoracic ventrally located intradural extramedullary meningiomas and schwannomas have been presented previously and include a unilateral transpedicular-transarticular approach. ${ }^{4}$ This provides ventral and lateral access to the thoracic spinal cord, which reduces spinal cord manipulation compared with a midline posterior approach. ${ }^{4}$ However, such posterolateral approaches have not yet been widely adopted for thoracic tumors, and further studies of their feasibility and alternatives are indicated.

Calcification of intradural spinal tumors presents an additional challenge. ${ }^{5}$ Solid calcifications only occur in $1 \%-$ $5 \%$ of spinal meningiomas, but they are less susceptible to internal decompression during resection, and attempts to mobilize calcified tumors are more prone to excess spinal 
TABLE 1. Preoperative patient features

\begin{tabular}{|c|c|c|c|c|c|c|}
\hline Patient No. & Age (yrs) & Sex & Symptoms & Symptom Duration (mos) & Tumor Location & Radiographic Findings \\
\hline 1 & 67 & $\mathrm{~F}$ & $\begin{array}{l}\text { Falls, gait imbalance, low-back pain, } \\
\text { bilateral numbness in feet, rt leg } \\
\text { radicular pain }\end{array}$ & 24 & T6, T10 & Extensive calcifications \\
\hline 2 & 75 & $\mathrm{~F}$ & $\begin{array}{l}\text { Gait difficulties progressing to inability } \\
\text { to ambulate, bilateral leg weakness, } \\
\text { poor bladder control, constipation }\end{array}$ & 6 & T10-12 & 5 distinct tumors \\
\hline 3 & 47 & $\mathrm{~F}$ & Upper-back pain, shoulder discomfort & 7 & T2-3 & Extensive calcifications \\
\hline 4 & 62 & M & $\begin{array}{l}\text { Bilateral leg weakness, hyperreflexia, } \\
\text { urinary frequency }\end{array}$ & 1 & T10-T11 & $\begin{array}{l}\text { Incidentally noted intradural mass } \\
\text { following MVA }\end{array}$ \\
\hline 5 & 66 & $\mathrm{~F}$ & $\begin{array}{l}\text { Inability to walk, urinary retention, con- } \\
\text { stipation, progressive back pain }\end{array}$ & 3 & T9 & $\begin{array}{l}\text { T9 intradural lesion, T11 vertebral } \\
\text { body lesion, T11 compression } \\
\text { fracture, lung mass, intracranial } \\
\text { metastatic disease }\end{array}$ \\
\hline
\end{tabular}

MVA $=$ motor vehicle accident

cord traction and increased risk of poor neurological outcome. ${ }^{6-9}$ This subset of thoracic tumors necessitates a surgical approach that addresses the dual problem of access to the ventral intradural space and the need for en bloc resection of calcified lesions. In the following case series, we present 5 cases of ventrally located intradural tumors, 4 of which were approached via a unilateral transpedicular posterolateral approach as opposed to the traditional posterior durotomy. Technical considerations and advantages are discussed, as well as the indications for this technique in light of our experience across two institutions.

\section{Methods \\ Data Collection}

A retrospective review of all operative spinal ventral thoracic tumor cases at two academic medical centers between May 2017 and August 2020 identified 5 patients who underwent resection of ventrally situated intradural extramedullary spinal tumors. All patients were assessed preoperatively via full neurological examination, spinal MRI, and spinal CT imaging. This study was approved as a retrospective review by the Rhode Island Hospital IRB and it was declared exempt by the State University of New York (SUNY) Upstate IRB, which waived the need for informed consent.

\section{Operative Technique}

Patients were positioned prone and underwent a standard surgical approach to expose the osseous anatomy at the relevant surgical levels. Somatosensory evoked potentials and transcortical motor evoked potentials were monitored intraoperatively. Pedicle screws were placed in standard fashion proximally and distally, and the pedicles at the level of the tumors were cannulated contralaterally. Bilateral laminectomies and ipsilateral facetectomies and pediculectomies were performed at the level of the tumor. The dura mater was opened with a curved semilunar incision and reflected laterally. The tumors were directly visualized, freed from arachnoid adhesions and small vessel attachments, and removed en bloc. The remaining pedicle screws were then placed in the previously drilled holes and stabilized with rod fixation and bone graft.

\section{Summary of Cases}

Case details are summarized in Table 1.

\section{Patient 1}

A 67-year-old woman with a past surgical history of L4-5 transforaminal lumbar interbody fusion. The patient presented with 2 years of bilateral foot numbness, right radicular leg pain, low-back pain, and gait imbalance with falls. Her examination was pertinent for antalgic gait. MRI of the thoracic spine revealed 2 homogeneously enhancing, ventral intradural extramedullary masses at T6 and T10. A CT scan of the thoracic spine revealed that both masses were calcified. The T6 tumor was located eccentrically, so we performed a T6-7 laminectomy, partial T5 laminectomy, right T6-7 facetectomy, T7 pediculectomy, and T7 partial rib resection. The T10 tumor was located eccentrically to the left, so we performed a T9-10 laminectomy, left T9-10 facetectomy, T10 pediculectomy, and T10 partial rib resection. Both tumors were removed en bloc, and the right $\mathrm{T} 7$ nerve root was ligated due to extensive tumor infiltration. Bilateral instrumentation was placed from T4 to T11. Histological grading revealed both tumors to be WHO grade I meningiomas. The patient's postoperative course was uncomplicated; postoperative imaging revealed adequate resection of tumors and stable positioning of spinal instrumentation (Fig. 1).

\section{Patient 2}

A 75-year-old woman had a recent past medical history of transient ischemic attack and myocardial infarction. The patient presented with inability to ambulate after a progressive decline over the previous 6 months, 2 months of urinary incontinence, and recent-onset constipation. Her examination was pertinent for brisk reflexes and diminished strength and sensation in the lower extremities bilaterally. MRI of the thoracic spine revealed 5 ventrally 

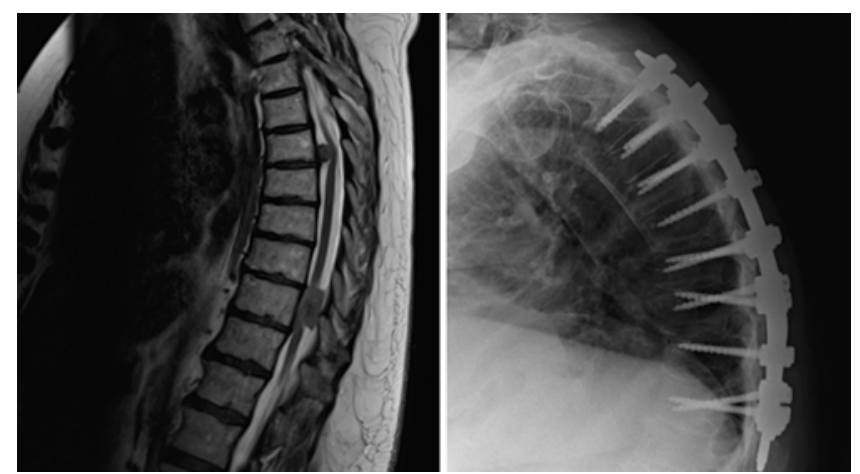

FIG. 1. Patient 1. Left: Preoperative sagittal MR image demonstrating 2 homogeneously enhancing, calcified, ventral intradural extramedullary masses located at T6 and T10. Right: Lateral plain radiograph obtained 3 months postoperatively, demonstrating stable positioning of spinal instrumentation from $\mathrm{T} 4$ to $\mathrm{T} 11$.

situated intradural extramedullary tumors from T10 to T12 that filled the spinal canal and severely compressed the spinal cord. The tumors were located eccentrically, so T9-12 laminectomies and right T10-12 facetectomies and pediculectomies were performed. All 5 tumors were removed with minimal retraction of the spinal cord or adjacent nerves. Bilateral instrumentation was placed from T9 to L1. Histological grading revealed all 5 tumors to be WHO grade I schwannomas. The patient's postoperative course showed no new neurological deficits. Postoperative imaging revealed adequate resection of all tumors and stable spinal instrumentation (Fig. 2; see Video 1, which demonstrates the utility of this approach).

VIDEO 1. Patient 2. Resection of 5 ventral intradural extramedullary tumors from T10-12 using a posterolateral transpedicular surgical approach. Copyright Michael A. Galgano. Published with permission. Click here to view.

\section{Patient 3}

A 47-year-old woman had a surgical history of L5-S1 decompression with posterior fusion. Follow-up imaging incidentally revealed a T2-3 ventral intradural extramedullary mass. The patient subsequently reported a history of upper-back pain and shoulder discomfort with pain radiating anteriorly, with no deficits on neurological examination. Preoperative imaging demonstrated extensive calcifications within the mass (Fig. 3). A T2-3 bilateral laminectomy was performed, and the dura was opened via a posterior longitudinal midline incision, without pediculectomy. The spinal cord herniated outward and a brief period of bradycardia was noted. Two lateral incisions were made in the dura, but attempts to establish a surgical plane led to decreased neuromonitoring signals bilaterally. Given the changes in neuromonitoring, no biopsy was performed and further attempts at resection were aborted. A T1 bilateral laminectomy was performed to maximally decompress the spinal cord. The patient's postoperative course revealed diminished sensation in the T1 distribution with normal strength and reflexes bilaterally.

The patient subsequently received external-beam radiation therapy to $\mathrm{T} 2-3$. Two years postoperatively, she

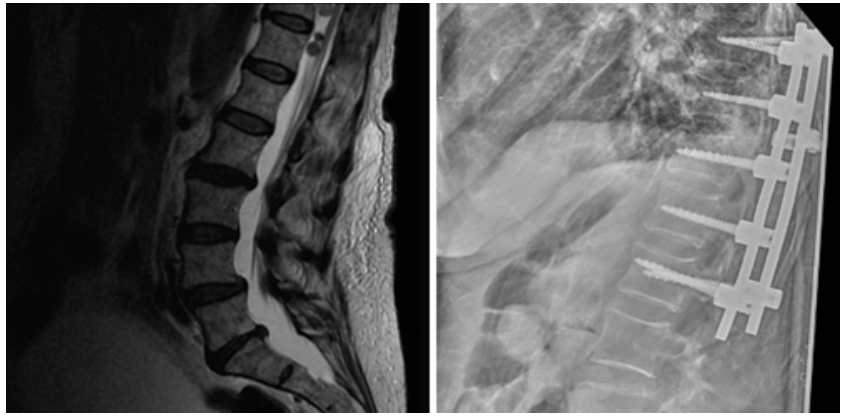

FIG. 2. Patient 2. Left: Preoperative sagittal MR image demonstrating 5 ventral intradural extramedullary tumors from T10 to T12 that fill the spinal canal with significant spinal cord compression. Right: A postoperative lateral plain radiograph demonstrates stable hardware positioning from T9 to L1.

reported left-sided tingling, several months of bilateral lower-extremity weakness, and persistent midback pain. Neurological examination showed intact function with no focal weakness or arm numbness bilaterally. Repeat thoracic MRI showed a stable-appearing tumor at T2-3 with some compression and stenosis of the cord. One month after this visit, the patient underwent a nerve block for conservative management of her back pain and developed acute-onset bilateral leg numbness that resolved in her right leg but persisted on the left, along with constipation. Repeat imaging again demonstrated a large, calcified mass with corresponding myelomalacia. The patient was brought to the operating room for extracavitary resection. The tumor was located eccentrically, so we performed a left T3 costotransversectomy and left T2 -3 and T3-4 facetectomies. The tumor was directly visualized and removed en bloc. Bilateral instrumentation was placed from T1 to T5. Histological grading revealed a calcified WHO grade I meningioma. The patient's postoperative course was complicated by fevers, likely due to chemical meningitis. Routine postoperative CSF sampling revealed gram-positive cocci, so empirical antibiotic coverage was added; all subsequent CSF samples were negative. At the time of discharge, the patient reported decreased pain and improved strength in her legs bilaterally (Fig. 3; see Video 2 , which demonstrates the importance of ventral spinal cord access).

VIDEO 2. Patient 3. Revision resection of a calcified ventral intradural extramedullary mass at T2-3 using an extracavitary approach. Copyright Michael A. Galgano. Published with permission. Click here to view.

\section{Patient 4}

A 62-year-old man presented after a minor car accident. CT imaging at the time incidentally revealed a T1011 ventral intradural extramedullary mass. On evaluation, the patient reported 1 month of progressive bilateral leg weakness and urinary frequency. Neurological examination was significant for diminished motor strength and hyperreflexia in bilateral lower extremities. The tumor was located eccentrically, so bilateral T10-11 laminectomies and right T10-11 facetectomies and pediculectomies were performed. The tumor was removed en bloc, and the right 


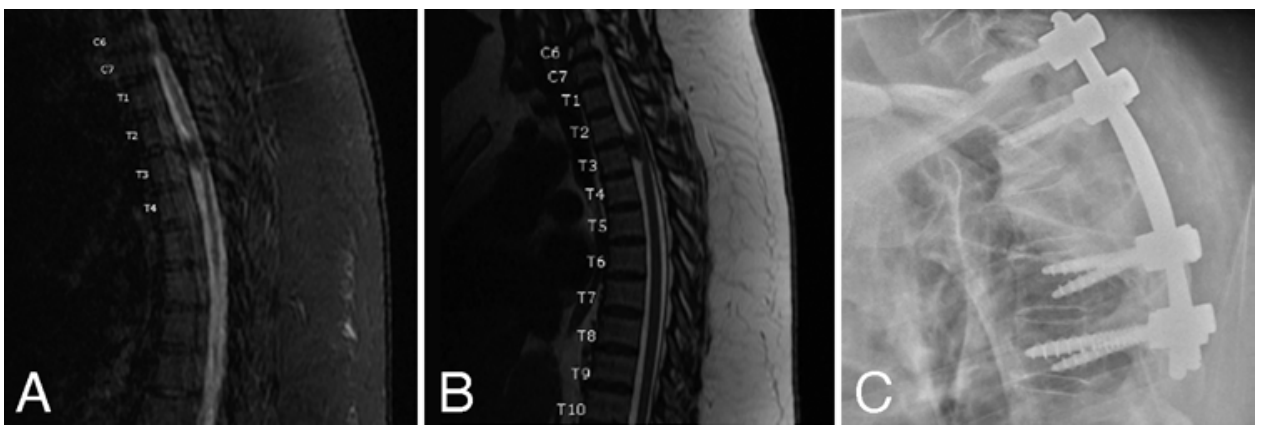

FIG. 3. Patient 3. A: Preoperative sagittal MR image obtained at the patient's original visit, demonstrating a T2-3 ventral intradural extramedullary mass with extensive calcifications throughout the mass. B: Repeat sagittal MR image showing interval progression of the calcified T2-3 mass with myelomalacia, spinal cord compression, and stenosis. C: Postoperative lateral plain radiograph demonstrating stable hardware positioning from $\mathrm{T} 1$ to $\mathrm{T} 5$.

T11 nerve motor root was ligated because of tumor infiltration. Bilateral instrumentation was placed from $\mathrm{T} 8$ to T12. Histological grading revealed WHO grade I schwannoma. At the 5-month follow-up visit, the patient had full strength and complete resolution of symptoms with stable hardware placement and interval fusion (Fig. 4).

\section{Patient 5}

A 66-year-old woman presented to the emergency department with a 3-day history of inability to ambulate, difficulty urinating and defecating, and 3 months of worsening back pain. MRI revealed a ventral intradural extramedullary T9 lesion with cord impingement and a T11 vertebral body lesion with an associated compression fracture, both initially concerning for metastatic lesions. An MRI scan of the brain revealed intracranial metastatic disease, and a CT scan of the chest identified a lung mass. Neurological examination was significant for bilateral clonus and lower-extremity weakness. Preliminary biopsy was concerning for non-small cell carcinoma. The tumor was eccentrically located, so we performed a bilateral T9 laminectomy, left T8-9 and T9-10 facetectomies, a left T9 pediculectomy, and a partial T8 laminectomy. We then completed T10-12 laminectomies, bilateral T10-12 facetectomies, bilateral T11 pediculectomies, and a T11
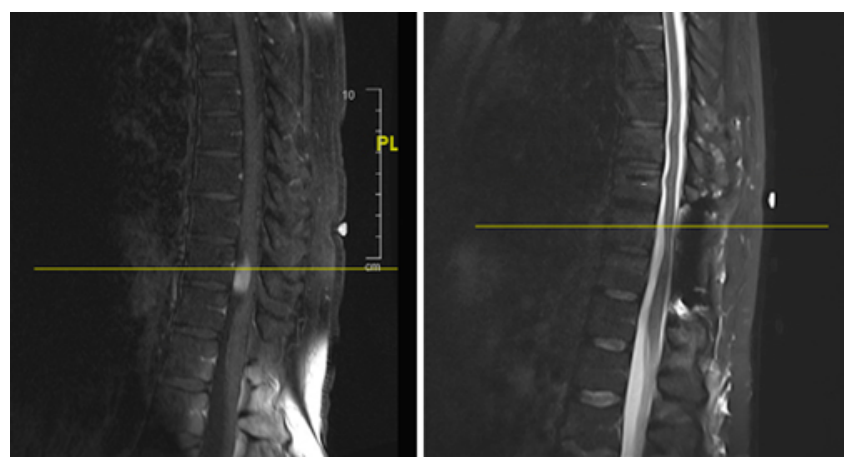

FIG. 4. Patient 4. Left: Preoperative sagittal MR image demonstrating a T10-11 ventral intradural extramedullary mass. Right: Sagittal MR image obtained 5 months postoperatively, demonstrating interval fusion with no evidence of tumor recurrence. transpedicular corpectomy. The T9 tumor was removed en bloc from the corpectomy cavity, followed by T10-11 and T11-12 discectomies with cage placement. Bilateral instrumentation was placed from T7 to L2. Histological grading of the T9 tumor revealed a WHO grade I meningioma with scattered calcifications, and the T11 lesion was consistent with metastatic lung adenocarcinoma. Postoperative imaging at 6 months revealed adequate resection of the T9 tumor and stable positioning of hardware with interval progression of the lung mass and osseous metastatic disease (Fig. 5). The patient has since died due to metastatic lung cancer.

\section{Discussion}

The differential diagnosis for intradural, extramedullary spinal tumors visualized on MRI is broad; however, meningiomas and schwannomas account for most of these lesions.$^{10}$ The typical patient is a middle-aged female who presents with focal back pain and progressive neurological symptoms over the course of 3 months to 2 years. ${ }^{11}$ Definitive management for symptomatic patients includes en bloc resection. Fortunately, large case series have suggested that complete resection is attainable in greater than $90 \%$ of patients, and only $5 \%-10 \%$ of these tumors are located ventral to the spinal cord. ${ }^{8,12,13}$ However, when ventral thoracic lesions do occur, they tend to be among the more surgically challenging intradural lesions and are associated with increased surgical morbidity due to numerous factors, including difficult thoracic anatomy, high thoracic cord-to-canal ratio, and suboptimal vascular supply to this region. ${ }^{5,14,15}$

The traditional surgical approach for an anterior, intradural, extramedullary lesion in the thoracic spine entails either a posterior approach with unilateral laminectomy and facetectomy, a lateral extracavitary approach, or costotransversectomy. ${ }^{16}$ Posterior microsurgical techniques have been refined over time but still require significant manipulation of the spinal cord for adequate tumor resection, which can lead to complications, including CSF leak and myelopathy. ${ }^{7,17,18}$ In the setting of ventral lesions, the posterior approach limits tumor exposure. ${ }^{19}$ Without pediculectomy, the surgical corridor around the cord is narrow, and the presence of complicating factors, including 

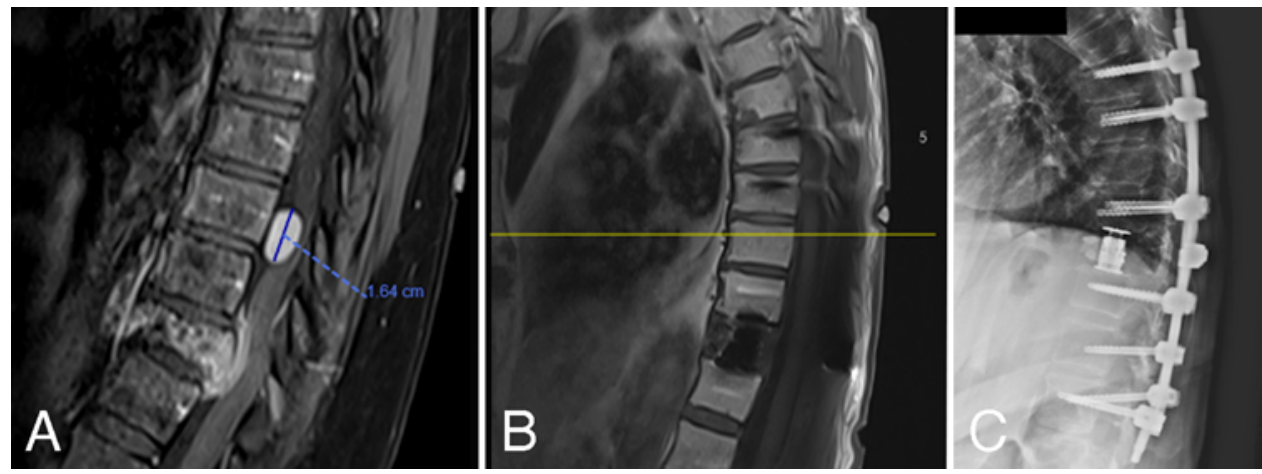

FIG. 5. Patient 5. A: Preoperative sagittal MR image demonstrating a ventral intradural extramedullary T9 lesion with cord impingement and a T11 vertebral body lesion with an associated compression fracture. B: Sagittal MR image obtained at the 6-month follow-up, demonstrating stable hardware positioning with interval progression of the lung mass and osseous metastatic disease. C: Lateral plain radiograph obtained at the 6-month follow-up, demonstrating stable hardware positioning from T7 to L2.

calcifications and adhesions, can further limit successful posterior resection..$^{19}$ Calcifications predispose patients to surgical complications and incomplete resection because they make it difficult to identify a surgical plane between the tumor and the spinal cord. ${ }^{5}$ For example, patient 3 had an extensively calcified ventral tumor and initially underwent a traditional posterior approach. Her case was terminated due to cord herniation and inability to identify a safe surgical plane. We posit that while the standard posterior approach applied initially for this patient was ultimately inadequate for successful resection, the transpedicular approach applied in the other 4 cases could have improved initial access to the tumor, resulting in complete resection and improved outcomes.

Our case series highlights the efficacy of the posterolateral transpedicular approach in the setting of complicated ventral intradural extramedullary pathology. The 4 patients in this series who underwent transpedicular resection of their intradural lesions had stable instrumentation and neurological examination findings on follow-up without recurrence of their lesions. Based on these cases, the posterolateral transpedicular approach improves on the posterior approach by providing a wider surgical corridor for access to the tumor. Unlike the posterior approach, this technique significantly decompresses the spinal cord with bilateral laminectomies and ipsilateral facetectomies and pediculectomies before manipulating the spinal cord and tumor, which reduces the risk of cord herniation and need for case termination. Additionally, this approach allows for direct visualization and easier identification of an appropriate surgical plane. Ultimately, patient 3 underwent a successful posterolateral extracavitary approach following interval progression, requiring a costotransversectomy and facetectomies, and she experienced a minor postoperative infection. This case redemonstrates the need for posterolateral access to the ventral canal in the setting of complex lesions, particularly those with extensive calcifications.

The posterolateral transpedicular approach has been validated for use in extradural pathology, such as ventral cervical tumors and vertebral body metastases with posterior epidural extension, but its use for ventral intradural thoracic lesions has been described infrequently.,
Acosta et al. described the benefit of a similar technique in patients with cervical and cervicothoracic intradural extramedullary spinal tumors, but this approach has not been well defined in thoracic tumors. ${ }^{9}$ The posterolateral approach limits the need for spinal cord retraction and rotation, providing the surgeon with wide access to the intradural lesion at the end of the transpedicular corridor. ${ }^{21}$ The cases we present further validate the feasibility and efficacy of other transpedicular techniques., ${ }^{4,21}$ Specifically, our surgical technique extends the ipsilateral facetectomy and pediculectomy technique of Ito et al. with the addition of multilevel bilateral laminectomies and posterior instrumentation. ${ }^{21}$ Our approach also expands on Acosta and colleagues' work with a new subset of tumors, and it validates the hypothesized benefit of their approach in cases of calcified tumors. ${ }^{9}$ We believe that posterior fixation reduces the risk of surgery-related misalignment and is especially important in patients who receive destabilizing multilevel resection of elements of the middle and posterior spinal columns. Thus, we advocate for the spine surgeon to consider additional decompression and instrumentation when planning a transpedicular route for ventral tumor resection.

While calcifications and ossifications represent only $1 \%-5 \%$ of spinal meningiomas, they are a challenging subset of these tumors. ${ }^{6}$ Tumor calcification and ventral location have both been independently identified as risk factors for poor postoperative outcomes and an increased likelihood of incomplete tumor resection. ${ }^{5,22}$ In this case series, 3 of 5 patients were found to have calcified or partially calcified intradural lesions. Our series demonstrates that the use of a transpedicular posterolateral approach allows for optimal resection of several particularly challenging intradural lesions, due to ventral extent or calcification. Patient 1 underwent a successful calcified tumor resection, and patient 2 underwent a successful resection of 5 ventral intradural extramedullary tumors, both via this posterolateral transpedicular approach. Patient 5 underwent a successful resection of an isolated meningioma in the setting of extensive adjacent spinal metastatic disease. This approach is versatile and allows for effective implementation in patients with complicated surrounding pathology. Review of additional patient cases is necessary to fully 
validate this approach in the setting of calcifications, but our initial results suggest a benefit to transpedicular posterolateral tumor resection in the setting of complex intradural ventral thoracic tumors.

\section{Study Limitations}

This retrospective study utilizes a small sample size and only includes 3 patients with radiographic and/or histological evidence of calcification. No statistical analysis could be performed to draw specific conclusions.

\section{Conclusions}

We evaluated the utility of a posterolateral transpedicular approach with posterior fixation for surgical treatment of ventral intradural extramedullary thoracic tumors. This study validates the surgical approach for carefully selected lesions across a small multiinstitutional patient sample. Furthermore, this study identifies a potential surgical option for the resection of complex, calcified spinal meningiomas.

\section{References}

1. Voulgaris S, Alexiou GA, Mihos E, et al. Posterior approach to ventrally located spinal meningiomas. Eur Spine J. 2010; 19(7):1195-1199.

2. Gambardella G, Gervasio O, Zaccone C. "Approaches and surgical results in the treatment of ventral thoracic meningiomas. Review of our experience with a postero-lateral combined transpedicular-transarticular approach." Acta Neurochir (Wien). 2003;145(5):385-392.

3. Raco A, Pesce A, Toccaceli G, et al. Factors leading to a poor functional outcome in spinal meningioma surgery: remarks on 173 cases. Neurosurgery. 2017;80(4):602-609.

4. Balaha AM, Shamhoot EA. Combined transpedicular-transarticular approach for ventrally located thoracic intradural meningiomas. Asian J Neurosurg. 2019;14(3):744-747.

5. Roux FX, Nataf F, Pinaudeau M, et al. Intraspinal meningiomas: review of 54 cases with discussion of poor prognosis factors and modern therapeutic management. Surg Neurol. 1996;46(5):458-464.

6. Xu F, Tian Z, Qu Z, et al. Completely ossified thoracic intradural meningioma in an elderly patient: a case report and literature review. Medicine (Baltimore). 2020;99(25):e20814.

7. Angevine PD, Kellner C, Haque RM, McCormick PC. Surgical management of ventral intradural spinal lesions. J Neurosurg Spine. 2011;15(1):28-37.

8. Sandalcioglu IE, Hunold A, Müller O, et al. Spinal meningiomas: critical review of 131 surgically treated patients. Eur Spine J. 2008;17(8):1035-1041.

9. Acosta FL Jr, Aryan HE, Chi J, et al. Modified paramedian transpedicular approach and spinal reconstruction for intradural tumors of the cervical and cervicothoracic spine: clinical experience. Spine (Phila Pa 1976). 2007;32(6):E203E210.

10. Traul DE, Shaffrey ME, Schiff D. Part I: Spinal-cord neoplasms-intradural neoplasms. Lancet Oncol. 2007;8(1): 35-45.

11. Saraceni C, Harrop JS. Spinal meningioma: chronicles of contemporary neurosurgical diagnosis and management. Clin Neurol Neurosurg. 2009;111(3):221-226.
12. Solero CL, Fornari M, Giombini S, et al. Spinal meningiomas: review of 174 operated cases. Neurosurgery. 1989;25(2): 153-160.

13. Maiti TK, Bir SC, Patra DP, et al. Spinal meningiomas: clinicoradiological factors predicting recurrence and functional outcome. Neurosurg Focus. 2016;41(2):E6.

14. Gezen F, Kahraman S, Canakci Z, Bedük A. Review of 36 cases of spinal cord meningioma. Spine (Phila Pa 1976). 2000;25(6):727-731.

15. Mehta AI, Adogwa O, Karikari IO, et al. Anatomical location dictating major surgical complications for intradural extramedullary spinal tumors: a 10-year single-institutional experience. J Neurosurg Spine. 2013;19(6):701-707.

16. Parsa AT, Lee J, Parney IF, et al. Spinal cord and intraduralextraparenchymal spinal tumors: current best care practices and strategies. J Neurooncol. 2004;69(1-3):291-318.

17. Riad H, Knafo S, Segnarbieux F, Lonjon N. Spinal meningiomas: surgical outcome and literature review. Neurochirurgie. 2013;59(1):30-34.

18. Sim JE, Noh SJ, Song YJ, Kim HD. Removal of intraduralextramedullary spinal cord tumors with unilateral limited laminectomy. J Korean Neurosurg Soc. 2008;43(5):232-236.

19. Kim CH, Chung CK. Surgical outcome of a posterior approach for large ventral intradural extramedullary spinal cord tumors. Spine (Phila Pa 1976). 2011;36(8):E531-E537.

20. Bilsky MH, Boland P, Lis E, et al. Single-stage posterolateral transpedicle approach for spondylectomy, epidural decompression, and circumferential fusion of spinal metastases. Spine (Phila Pa 1976). 2000;25(17):2240-2250.

21. Ito K, Aoyama T, Miyaoka Y, et al. Surgery for ventral intradural thoracic spinal tumors with a posterolateral transpedicular approach. Acta Neurochir (Wien). 2016;158(8): $1563-1569$.

22. Ruggeri AG, Fazzolari B, Colistra D, et al. Calcified spinal meningiomas. World Neurosurg. 2017;102:406-412.

\section{Disclosures}

Dr. Gokaslan reports being a topic editor for Neurosurgical Focus.

\section{Author Contributions}

Conception and design: Galgano. Acquisition of data: all authors. Analysis and interpretation of data: Visco, Liu, Leary. Drafting the article: Visco, Liu, Leary. Critically revising the article: all authors. Reviewed submitted version of manuscript: all authors. Statistical analysis: Camara-Quintana. Administrative/technical/ material support: Galgano, Oyelese, Gokaslan. Study supervision: Galgano, Oyelese, Gokaslan, Camara-Quintana.

\section{Supplemental Information \\ Videos}

Video 1. https://vimeo.com/518109981.

Video 2. https://vimeo.com/518110123.

Video Abstract. https://vimeo.com/530279938.

\section{Correspondence}

Michael A. Galgano: SUNY Upstate Medical University, Syracuse, NY.galganom@upstate.edu. 